\title{
GALLBLADDER PERFORATION AFTER ACALCULOUS CHOLECYSTITIS
}

\author{
Lokesh Kulshrestha ${ }^{1}$
}

${ }^{1}$ Associate Professor, Department of Surgery, S. N. Medical College, Agra, India.

HOW TO CITE THIS ARTICLE: Kulshrestha L. Gallbladder perforation after acalculous cholecystitis. J. Evolution Med. Dent. Sci. 2017;6(94):6918-6919, DOI: $10.14260 /$ jemds/2017/1500

\section{PRESENTATION OF CASE}

A sixty two year old female was admitted in the surgical unit of the hospital with complaints of pain in epigastrium and distension of abdomen for last 2 days. Pain was initially localised, but became generalised with the passage of time. There was no history of fever and vomiting and no other associated symptoms. There was no past history suggestive of cholelithiasis. On examination, patient was moderately dehydrated. Her BP was $100 / 70 \mathrm{mmHg}$, pulse rate was $120 / \mathrm{mt}$, low in volume but regular, and respiratory rate was 24/mt shallow. Abdomen was distended with generalised tenderness on palpation and bowel sounds were absent. The systemic examination was within normal limits.

Gallbladder perforation (GBP) with chemical peritonitis is a rare but life-threatening condition, which usually requires immediate surgical intervention. Most cases are the complication of acute cholecystitis associated with cholelithiasis, although acute acalculous cholecystitis or intramural vessel thrombosis can sometimes lead to gallbladder perforation.

\section{DIFFERENTIAL DIAGNOSES}

a. Bacterial Peritonitis after duodenal perforation.

b. Chemical Peritonitis after gallbladder/ CBD perforation.

\section{CLINICAL DIAGNOSIS}

Ultrasonography of abdomen revealed small amount of collection of clear fluid without septation and gallbladder margins were not very clearly identifiable. The $x$-ray abdomen erect view revealed no signs of intestinal obstruction or pneumoperitoneum.

Blood examination showed a white blood cell count of $10900 / \mathrm{mm}^{3}$, total bilirubin level of $1.2 \mathrm{mg} / \mathrm{dL}$, SGPT of 47 $\mathrm{IU} / \mathrm{L}$, serum amylase level of $338 \mathrm{IU} / \mathrm{L}$ and serum lipase level of $155 \mathrm{IU} / \mathrm{L}$.

Initially, the patient was treated on the line of conservative management with fluid resuscitation, supportive treatment and antibiotics with the probable diagnosis of acute cholecystitis. However, there was no relief in abdominal pain and abdominal distension also increased and repeat ultrasonography showed moderate amount of collection in abdomen. So, a perforation peritonitis was suspected.

Then, laparotomy was performed the day after admission. On laparotomy, abdomen was full of bile stained fluid with partial necrotic changes in neck and body of the gall bladder.

'Financial or Other Competing Interest': None.

Submission 29-10-2017, Peer Review 25-11-2017,

Acceptance 01-12-2017, Published 18-12-2017.

Corresponding Author:

Lokesh Kulshrestha,

\#23, Suresh Nagar,

Agra-282005 India.

E-mail:lokesh@doctor.com

DOI: $10.14260 /$ jemds $/ 2017 / 1500$

(c) $(1) \odot$
Cholecystectomy was performed and abdominal cavity was washed thoroughly with copious amounts of warm normal saline. There was no calculus seen anywhere. Postoperative period was uneventful.

\section{PATHOLOGICAL DISCUSSION}

Pathological examination of the resected gallbladder revealed inflammatory changes of its wall. There was no evidence of cholelithiasis. Microbiological test of bile showed negative results.

\section{DISCUSSION OF MANAGEMENT}

Niemeier, in 1934, classified perforation of the gallbladder into three types: Type 1, Acute Free Perforation (with generalised peritonitis); Type 2, Subacute Perforation (with abscess formation); and Type 3, Chronic Perforation (with fistula formation).[1] The gallbladder perforation is due to inflammation, trauma or obstruction.

Perforation of the gallbladder can be classified into three groups: [A] Spontaneous, [B] Traumatic and [C] Iatrogenic. The spontaneous group is further sub-divided into an idiopathic group and a secondary group which includes acute inflammation, infection, lithiasis, congenital obstruction and anticoagulant therapy etc.

In acute cholecystitis, inflammation causes ischaemic changes in the gallbladder wall leading to gangrene and perforation. This might explain why most cases of gallbladder perforation follow an exacerbation of acute cholecystitis with cholelithiasis, and gallbladder perforation without gallstones is rare. Such cases are mainly attributed to impairment of the blood supply induced by intramural thrombosis. So, idiopathic spontaneous perforation of acalculous gallbladder is very rare.

Strohl EL et al[2] reported the incidence of gallbladder perforation to be $9.6 \%$ among cholecystectomised patients in a series involving 31 patients whose symptoms were similar to those in patients with uncomplicated acute cholecystitis.

According to Bedirli A et al[3] 12 (3.3\%) cases of acute cholecystitis were complicated by gall bladder perforation out of a total of 386 patients, while Menakuru SR et al[4] reported the incidence of gall bladder perforation complicating acute cholecystitis in $5.9 \%$ of 31 patients.

The gallbladder perforation in this case presumably occurred within the first 24 hours of the onset of symptoms. Williams NF (1976) ${ }^{[5]}$ reported that perforation occurs most commonly within 72 hours.

The gallbladder perforation occurs most commonly at fundus due to least blood supply,[2] while in this case the perforation occurred at the neck of the gallbladder which is an uncommonly reported site for such an event.

Clinical diagnosis of idiopathic spontaneous gallbladder perforation is very difficult. Confirmatory diagnosis is only after laparotomy and by seeing bile coloured peritoneal collection and perforated gallbladder. 
Our case is unusual, because our patient had no prior history suggestive of gallbladder disease and had no known medical comorbidity.

\section{FINAL DIAGNOSIS}

In case of peritonitis, an option of chemical peritonitis should be kept in differential diagnosis. Gallbladder perforation is rarely diagnosed preoperatively. Although, with a high index of suspicion, awareness and diagnostic procedures can help in preoperative diagnosis. Ultrasound scan and CT scan may not help in early diagnosis. However, final diagnosis is usually confirmed at laparotomy. Interval cholecystectomy in all diagnosed cases of acalculous cholecystitis should be a viable option to consider preventing future complications as emphasised by this case.

\section{REFERENCES}

[1] Niemeier OW. Acute free perforation of the gallbladder. Ann Surg 1934;99(6):922-4.

[2] Strohl EL, Diffenbaugh WG, Baker JH, et al. Collective reviews: gangrene and perforation of the gallbladder. Int Abstr Surg 1962;114:1-7.

[3] Bedirli A, Sakrak O, Sözüer EM, et al. Factors effecting the complications in the natural history of acute cholecystitis. 2001;48(41):1275-8.

[4] Menakuru SR, Kaman L, Behera A, et al. Current management of gall bladder perforations. ANZ J Surg 2004;74(10):843-6.

[5] Williams NF, Scobie TK. Perforation of the gallbladder: analysis of 19 cases. Can Med Assoc J 1976;115(12):1223-5. 